\title{
Expression of matrix metalloproteinase-7 correlates with the invasion of $T 1$ colorectal carcinoma
}

\author{
FUMIHIKO URUSHIBARA $^{1}$, EISUKE SHIOZAWA ${ }^{1}$, HIDEYUKI MIYACHI ${ }^{2}$, MASASHI MISAWA ${ }^{2}$, \\ TOMONARI CHO $^{1}$, YUSUKE TAKEHARA ${ }^{1}$, NANA ARAI ${ }^{1}$, TOSHITAKA FUNAKI ${ }^{1}$, SAKIKO TAZAWA ${ }^{1}$, \\ MAYUMI HOMMA ${ }^{1}$, TOMOKO NOROSE ${ }^{1}$, MUTSUKO OMATSU ${ }^{3}$, TOSHIKO YAMOCHI ${ }^{1}$, TOSHIAKI KUNIMURA ${ }^{3}$, \\ GENSHU TATE $^{1}$, KAZUHO HONDA ${ }^{4}$, ISHIDA FUMIO ${ }^{2}$, SHIN-EI KUDO ${ }^{2}$ and MASAFUMI TAKIMOTO ${ }^{1}$ \\ ${ }^{1}$ Department of Pathology, Showa University School of Medicine, Tokyo 142-8555; \\ ${ }^{2}$ Digestive Disease Center; ${ }^{3}$ Department of Diagnostic Pathology, Showa University Northern Yokohama Hospital; \\ ${ }^{4}$ Department of Anatomy, Showa University School of Medicine, Tokyo 142-8555, Japan
}

Received October 3, 2016; Accepted March 3, 2017

DOI: $10.3892 / \mathrm{ol} .2018 .7746$

\begin{abstract}
T1 colorectal carcinomas (CRCs) are an initial site of metastatic spread. Various risk factors for lymph node metastasis have been investigated in T1 CRCs. However, the major step in the entire process of metastasis remains unclear. In terms of carcinoma invasion and metastasis, matrix metalloproteinases (MMPs) have recently gained increasing attention. Notably, MMP-7 is frequently overexpressed in CRCs, but its implication has not been determined in T1 CRCs yet. The present study aimed to clarify the associations between the pathological risk factors of T1 CRCs and MMP-7. In the current study, 211 lesions of T1 CRC that were resected endoscopically or surgically at Showa University Northern Yokohama Hospital (Yokohama, Japan) between April 2008 and December 2009 were retrospectively analyzed. MMP-7 was immunostained and evaluated by its frequency of expression. Pathological factors of T1 CRCs were analyzed in association with MMP-7 expression. Furthermore, the ultrastructural alterations of carcinoma invasion were examined using low vacuum-scanning electron microscopy (LV-SEM). MMP-7 expression was associated with venous invasion $(\mathrm{P}=0.005)$, and LV-SEM revealed the disappearance of the normal structure of collagen and elastic fibers of veins invaded by tumor cells expressing MMP-7. At the invasive front, MMP-7 has a vital role in carcinoma invasion, correlating with venous invasion of T1 CRCs.
\end{abstract}

Correspondence to: Dr Fumihiko Urushibara, Department of Pathology, Showa University School of Medicine, 1-5-8 Hatanodai, Shinagawa-ku, Tokyo 142-8555, Japan

E-mail: urufumi1117@yahoo.co.jp

Key words: low vacuum-scanning electron microscopy, matrix metalloproteinase-7, venous invasion, T1 colorectal cancer, risk factor

\section{Introduction}

With the recent advances in endoscopic treatment, numerous T1 colorectal carcinomas (CRCs) are resected endoscopically with negative margins (1), and the proportion of early carcinomas amenable to endoscopic resection has increased (2). Previously identified risk factors for lymph node metastasis in T1 CRCs include lymphovascular invasion, histological grade, tumor budding, poor differentiation and degree of submucosal invasion (3-5). However, the mechanism of these risk factors and the molecular markers associated with carcinoma cell invasion remain to be fully elucidated in T1 CRCs.

Matrix metalloproteinases (MMPs) are a family of $\mathrm{Zn}^{2+}$-dependent proteolytic enzymes involved in the physiological and pathological remodeling of the extracellular matrix (ECM) during proliferation, angiogenesis and wound healing (6). MMP-7 is the smallest metalloproteinase identified to date (6), and has important roles in the degradation of ECM proteins (including fibronectin, laminin and collagen IV) and the regulation of several biochemical processes such as activation, degradation and shedding of non-ECM proteins (7). MMP-7 has attracted attention because previous studies detected overexpression of MMP-7 in invasive cancers of digestive organs such as the esophagus, stomach, colon and liver (7). In addition, overexpression of MMP-7 has been revealed in cancers of other organs, including the lungs, skin, breast, prostate, head and neck (7). A previous immunohistochemical study (8) revealed a significant correlation between MMP-7 expression at the invasive front and metastasis or prognosis of advanced CRCs. However, few studies have focused on T1 CRCs, and no definite conclusions could be obtained about the implication of MMP-7 with reference to pathological factors.

In the present study, the ability to examine three-dimensional ultrastructural changes of carcinoma invasion using low vacuum-scanning electron microscopy (LV-SEM) was identified, in addition to routine observation by light microscopy. To the best of our knowledge, no study has used this new technique to investigate the morphological and qualitative alterations of the ECM in CRCs to date. LV-SEM may be able 
to evaluate three-dimensional ultrastructural changes of the ECM using the same sections used for light microscopy, which would be considered as a novel and useful tool to assess the condition of the carcinoma invasion front.

Considering the aspects described above, the present study aimed to clarify the association of MMP-7 expression with pathological factors of T1 CRCs using immunohistochemistry, and to examine the three-dimensional ultrastructural changes of carcinoma invasion using LV-SEM.

\section{Materials and methods}

Patients and clinical data. Between April 2008 and December 2009, 211 consecutive cases of T1 CRCs were resected endoscopically or surgically at Showa University Northern Yokohama Hospital (Yokohama, Japan). Written informed consent was obtained from all patients prior to endoscopy or surgery, and the study design was approved by the Ethical Review Committee of Showa University School of Medicine Showa (Showa, Japan; approval no. 1929). Patients who were diagnosed with ulcerative colitis $(n=1)$ were excluded from the study. Pathological evaluation was not possible for 21 patients due to damage to or loss of the specimen. In total, 189 cases underwent only endoscopic treatment $(n=52)$, initial surgery $(n=57)$ or additional surgery $(n=80)$ with nodal dissection. These procedures were performed in accordance with the principles of the Japanese Society for Cancer of the Colon and Rectum (JSCCR) guidelines (9). The age and sex of the patients, and the location and size of the lesions, were reviewed from the electronic records system. The endoscopic morphology of the lesion was classified as 'depressed', 'flat' or 'protruded-type' based on the Kudo's morphological/development classification (10).

Pathological evaluations. All resected lesions were retrieved and immediately fixed in $10 \%$ buffered formalin for 24-48 h. Subsequently, the resected lesions were cut at the point where the deepest invasion area could be exposed on the cut-end surface. The serial histological specimens were cut into parallel 2-3 mm-thick slices. The tumor size was measured upon formalin fixation. The specimens were examined based on the 2010 World Health Organization (WHO) criteria (11) and JSCCR guidelines (9). The histological grade, tumor budding, desmoplastic reaction (DR) and growth type were investigated using hematoxylin and eosin (H\&E)-stained specimens. Lymphatic invasion was evaluated using $H \& E$ staining and immunostaining with an anti-D2-40 antibody (Clone D2-40; cat. no. IR072; dilution, ready to use; Dako; Agilent Technologies, Inc., Santa Clara, CA, USA), with which the samples were incubated for $15 \mathrm{~min}$ at room temperature. Venous invasion was evaluated using H\&E and Victoria Blue B staining. The histological grade was based on the least-differentiated tumor component and classified according to the 2010 WHO criteria (11). In the present study, a poorly differentiated adenocarcinoma or mucinous carcinoma component was considered present if any part of the lesion contained either of these features. Tumor budding was defined as an isolated single cancer cell or a cluster of $<5$ carcinoma cells at the invasive front (12). The field where budding was most intensive was selected to count the number of buds with a 20X-objective lens. Fields with $\geq 5$ buds were considered 'positive' according to Ueno et al (13). The growth type was evaluated as polypoid or non-polypoid growth according to Shimoda et al (14). The vertical invasion depth was measured based on the JSCCR guidelines (9). The degree of submucosal invasion was evaluated according to the Kudo's classification of degree of submucosal invasion $(15,16)$. The status of the muscularis mucosae (i.e., MM grade) was evaluated using desmin immunostaining (clone D33; cat. no. IR606; dilution ready to use; Dako; Agilent Technologies, Inc.) with which the samples were incubated for $15 \mathrm{~min}$ at room temperature, and was classified as 'grade 1' when the muscular fibers were maintained or as 'grade 2' when the muscle fibers had fragmented or disappeared (17).

Immunohistochemistry of MMP-7. Serial sections (4- $\mu \mathrm{m}$ thick) were newly cut from the slice with the deepest invasion area. The sections were stained using an automated immunohistochemistry staining device (BOND-III; Leica Microsystems, Inc., Buffalo Grove, IL, USA). In brief, the $4 \mu \mathrm{m}$-thick sections were transferred onto poly-L-lysine-coated adhesive slides and dried at $62^{\circ} \mathrm{C}$ for $30 \mathrm{~min}$. The sections were then incubated with the primary anti-MMP-7 antibody (clone ID-2; cat. no. M0785; dilution 1:2,000; Merck KGaA, Darmstadt, Germany) with which the samples were incubated for $15 \mathrm{~min}$ at room temperature, using a Bond Polymer Refine Detection kit (Leica Biosystems Newcastle, Newcastle, UK).

Evaluation of MMP-7 expression. MMP-7 is unique because its expression is restricted to tumor cells, and can be stained in the cytoplasm and cell membranes (Fig. 1) (8). In accordance with the procedure used in previous studies (18-21), MMP-7 immunostaining signals were counted in five fields at the invasive front. Scores were calculated as the percentage of tumor cells expressing MMP-7 divided by the total number of carcinoma cells in each field. The specimens were then classified as follows: 'Negative' (-); 'low expression' for $<10 \%$ cells expressing MMP-7 (1+); 'moderate expression' for $10-50 \%$ cells expressing MMP7 (2+); 'high expression' for $50-80 \%$ cells expressing MMP-7 (3+); and 'intense expression' for $>80 \%$ cells expressing MMP-7 (4+). Tumor cells expressing MMP-7 were considered as 'positive' if the median of the scores in the five fields was $3+$ or $4+$. Representative findings are shown in Fig. 2.

Immunohistochemistry of collagen IV. Collagen IV is the principal structural component of the ECM in the vascular basement membrane (22). In the present study, to examine the morphological and qualitative alterations of the injured vascular basement membrane using immunostaining for collagen IV, a number of MMP-7-positive samples were selected from the 189 cases of T1 CRC. As a normal control, areas where the carcinoma had not invaded to the submucosa were also prepared. Tissue sections (4- $\mu \mathrm{m}$ thick) from MMP-7-positive cases and normal controls were stained with an anti-collagen IV antibody (clone CIV22; cat. no., MAB13414; dilution 1:50; Dako; Agilent Technologies, Inc.) with which the samples were incubated for $15 \mathrm{~min}$ at room 


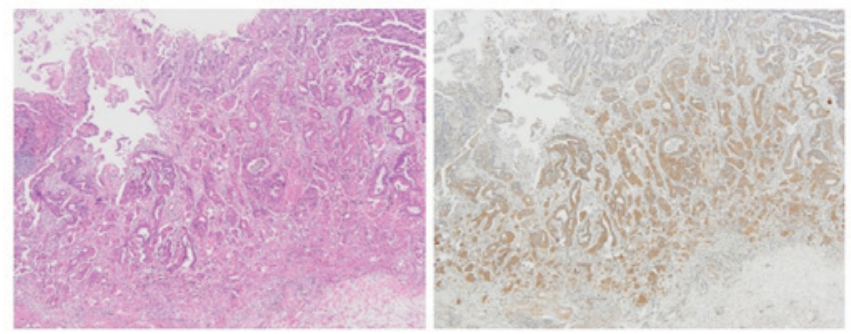

Figure 1. Expression of MMP-7 at the invasive front of T1 colorectal carcinomas. Panoramic view (magnification, x20) of (left) hematoxylin and eosin stain and (right) immunohistochemistry of MMP-7 of a representative section. The cytoplasm and cell membrane of tumor cells were stained for MMP-7, while stromal cells other than a few monocytes or surrounding normal mucosa remained unstained. MMP-7, matrix metalloproteinase-7.
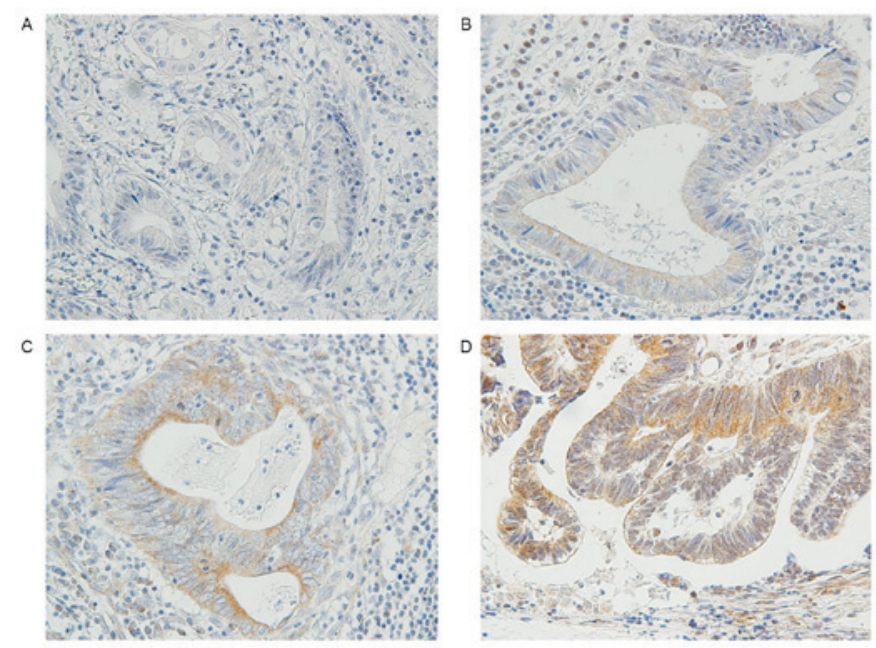

$\mathrm{E}$

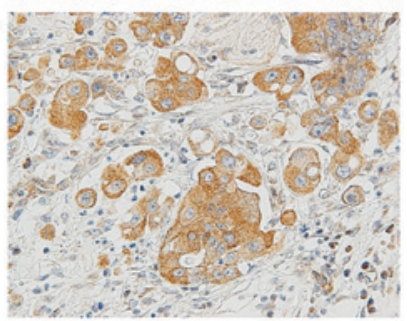

Figure 2. Immunohistochemical analysis of MMP-7 in T1 colorectal carcinomas. MMP-7-expressing cells were counted at the invasive front, and scores were calculated as the number of stained cells divided by the total number of carcinoma cells at the invasive front. The scores were classified as follows: (A) 'Negative expression' (-) (magnification, $\mathrm{x} 400$ ); (B) 'low expression' (+) (magnification, x200); (C) 'moderate expression' (2+) (magnification, x200); (D) 'high expression' (3+) (magnification, $x 200$ ); and (E) 'intense expression' (4+) (magnification, $x 400$ ). Immunostaining of MMP-7 was considered as 'positive' if the median of the scores in five fields at the invasive front was $3+$ or 4+. MMP-7, matrix metalloproteinase-7.

temperature using the aforementioned BOND-III automated immunohistochemistry staining device and a Bond Polymer Refine Detection kit. The positive staining (brown) of collagen IV was identified in vessel walls.

$L V$-SEM. Sections with Masson's trichrome staining were examined without a mounting coverslip under LV-SEM using a tabletop microscope (TM3000; Hitachi Ltd., Tokyo, Japan). The present study compared the same area in MMP-7-positive samples and normal controls using serial sections (4- $\mu \mathrm{m}$ thick)
Table I. Clinicopathological factors of 189 CRC cases.

Clinicopathological factors

Age $($ mean $\pm \mathrm{SD})$, years

Sex

Female

Male

Tumor size (mean $\pm \mathrm{SD})$, mm

Location

Rectum

Colon

Tumor budding

$+$

Lymphatic invasion

$+$

$-$

Venous invasion

$+$

Por/Muc component

$+$

Growth type

PG

NPG

Morphology

Depressed

Non-depressed

DR on the superficial layer

$+$

Depth of invasion, $\mu \mathrm{m}$

$<1,000$

$\geq 1,000$

Kudo's classification of degree of submucosal invasion

sm1a, $1 \mathrm{~b}$

sm1c, 2, 3

151

MM grade

2

Depth of invasion (mean $\pm \mathrm{SD}), \mu \mathrm{m}$

CRC, colorectal carcinoma; DR, desmoplastic reaction; MM, muscularis mucosae; Muc, mucinous carcinoma; NPG, non-polypoid growth; PG, polypoid growth; Por, poorly differentiated adenocarcinoma; SD, standard deviation.

subjected to immunostaining for collagen IV and stained with Masson's trichrome staining for observation under LV-SEM. During the staining process, $10-\mu \mathrm{m}$ thick sections 
Table II. Association between various pathological factors and MMP-7 expression.

\begin{tabular}{|c|c|c|c|c|}
\hline \multirow[b]{2}{*}{ Pathological factors } & \multicolumn{2}{|c|}{$\begin{array}{c}\text { MMP-7 } \\
\text { expression at the invasive front }\end{array}$} & \multirow[b]{2}{*}{ OR $(95 \% \mathrm{CI})$} & \multirow[b]{2}{*}{ P-value ${ }^{a}$} \\
\hline & Positive ( $\mathrm{n}=104)$ & Negative $(\mathrm{n}=85)$ & & \\
\hline Tumor budding & & & $1.20(0.45-3.38)$ & 0.821 \\
\hline+ & 13 & 9 & & \\
\hline- & 91 & 76 & & \\
\hline Lymphatic invasion & & & $1.16(0.56-2.43)$ & 0.733 \\
\hline+ & 26 & 19 & & \\
\hline- & 78 & 66 & & \\
\hline Venous invasion & & & $2.67(1.29-5.74)$ & 0.005 \\
\hline+ & 38 & 15 & & \\
\hline- & 66 & 70 & & \\
\hline Por/Muc component & & & $0.85(0.36-1.99)$ & 0.697 \\
\hline+ & 16 & 15 & & \\
\hline- & 88 & 70 & & \\
\hline Growth type & & & $1.24(0.65-2.37)$ & 0.540 \\
\hline PG & 70 & 53 & & \\
\hline NPG & 34 & 32 & & \\
\hline Morphology & & & $1.48(0.66-3.45)$ & 0.350 \\
\hline Depressed type & 22 & 13 & & \\
\hline Non-depressed type & 82 & 72 & & \\
\hline DR on the superficial layer & & & $1.18(0.53-2.66)$ & 0.712 \\
\hline+ & 21 & 15 & & \\
\hline- & 83 & 70 & & \\
\hline Depth of invasion, $\mu \mathrm{m}$ & & & $1.00(0.49-2.01)$ & 1.000 \\
\hline$<1,000$ & 27 & 22 & & \\
\hline$\geq 1,000$ & 77 & 63 & & \\
\hline Kudo's classification of degree of submucosal invasion & & & $1.13(0.52-2.45)$ & 0.856 \\
\hline sm1a, $1 \mathrm{~b}$ & 20 & 18 & & \\
\hline sm1c, 2,3 & 84 & 67 & & \\
\hline MM grade & & & $1.52(0.59-4.00)$ & 0.383 \\
\hline 2 & 93 & 72 & & \\
\hline 1 & 11 & 13 & & \\
\hline
\end{tabular}

${ }^{\text {aS }}$ Statistical analyses were performed using the Fisher's exact tests. CI, confidence interval; DR, desmoplastic reaction; MM muscularis mucosae; MMP-7, matrix metalloproteinase-7; Muc, mucinous carcinoma; NPG, non-polypoid growth; OR, odds ratio; PG, polypoid growth; Por, poorly differentiated adenocarcinoma.

were firstly stained with the anti-MMP-7 antibody visualized by 3,3'-Diaminobenzidine and incubated for $15 \mathrm{~min}$ at room temperature, then secondly stained with Victoria Blue B for light microscopy examination. Upon demounting, these sections were stained with Masson's trichrome staining for LV-SEM observation.

Statistical analysis. The association between MMP-7 expression and the pathological factors mentioned above was examined using the Fisher's exact test. $\mathrm{P}<0.05$ was considered to indicate a statistically significant difference. All analyses were conducted using R statistical software version 2.10.0 (R Foundation, Vienna, Austria; available at http://www.r-project.org).

\section{Results}

Clinical characteristics. As shown in Table I, endoscopic or surgical treatment was administered to 189 patients, of which 126 were male and 63 were female. The patients had a mean age of 66.0 \pm 10.9 years (range, $31-89$ years). The mean tumor size was 21.0 $\pm 11.5 \mathrm{~mm}$ (range, 4-65 mm). There were 35 depressed-type, and 154 flat and protruded-type lesions. In total, 143 lesions were in the colon and 46 lesions were in the rectum. The depth of invasion was 3,078.0 2 2,994.4 $\mu \mathrm{m}$ (range, 0-21,200 $\mu \mathrm{m}$ ).

Association of MMP-7 expression with pathological factors. The expression of MMP-7 was positive in 104 (55.0\%) of the 

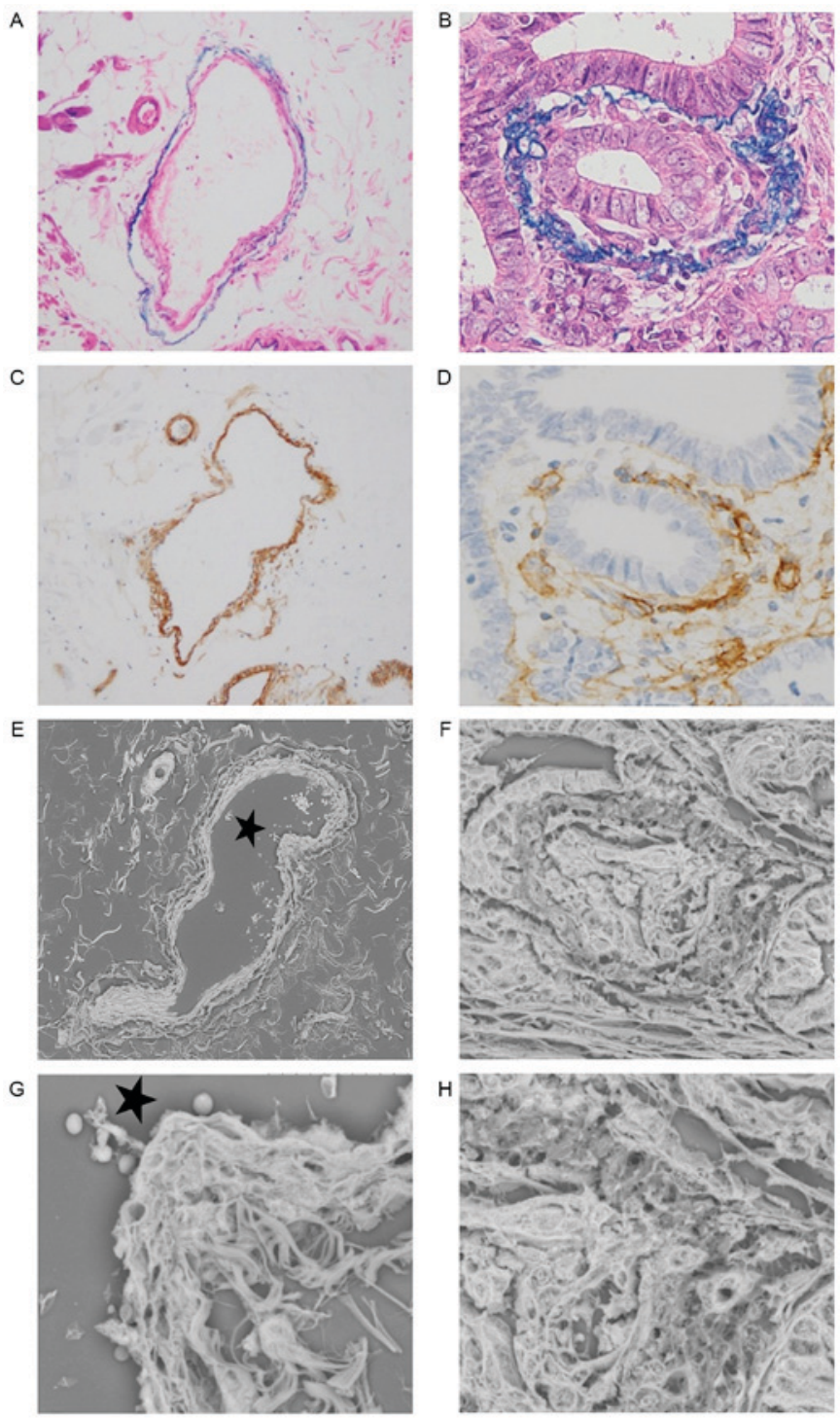

Figure 3. Histological findings of (A and B) hematoxylin and eosin, Victoria Blue $B$ and $(C$ and $D)$ collagen IV staining, and $(\mathrm{E}-\mathrm{H})$ three-dimensional ultrastructural findings in LV-SEM. Panels A, C, E and G show a normal vein in serial sections, while panels $\mathrm{B}, \mathrm{D}, \mathrm{F}$ and $\mathrm{H}$ show a vein invaded by tumor cells with positive matrix metalloproteinase-7 expression in serial sections. (A) Victoria Blue B staining was specifically visible in the elastic fibers of the adventitia (magnification, x200). (B) Victoria Blue B staining of adventitia was thinner on the invasion side (magnification, $x 400$ ). (C) Collagen IV staining was visible in the intima. Collagen IV of the normal vein was stained as a strong and thick linear pattern (magnification, $\mathrm{x} 200$ ). (D) Collagen IV staining of the intima was weak and interrupted by the invading tumor cells (magnification, x200). (E) Serial section stained with phosphotungstic acid (Masson's trichrome staining) and assessed by LV-SEM (magnification, $\mathrm{x} 300$ ). (F) Veins invaded by tumor cells were characterized by an irregular change in the two vascular layers in LV-SEM (magnification, x800). (G) A normal vein was characterized by a two-layered structure with smooth elastic fibers of the adventitia and a mesh-like structure of collagen fibers in the intima by LV-SEM (magnification, $\mathrm{x} 1,500)$. $(\mathrm{H})$ The thin portion of collagen IV staining was characterized by disappearance of the intimal mesh-like structure of collagen fibers in LV-SEM (magnification, x1,500). The invading tumor cells in an isolated and clustered form were visible within the altered venous walls in LV-SEM (magnification, $x 1,500$ ). The stars in $(E)$ and $(G)$ indicate the same lumen. LV-SEM, low vacuum-scanning electron microscopy.

189 examined lesions. As shown in Table II, MMP-7 expression was positive in $38(71.7 \%)$ of 53 lesions with venous invasion and in $66(48.5 \%)$ of 136 lesions without venous invasion. This
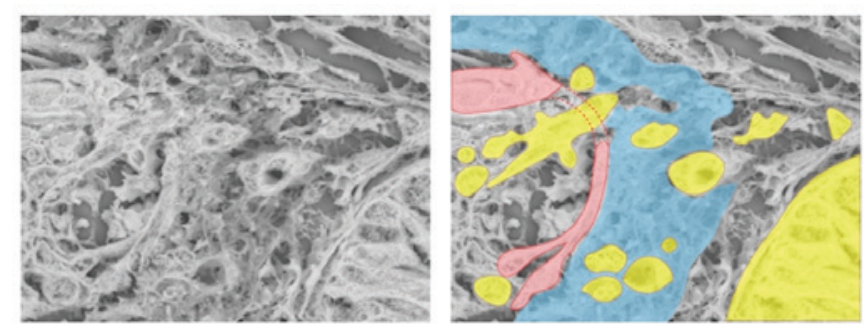

Figure 4. Left panel represents ultrastructural findings of a vein invaded by tumor cells in low vacuum-scanning electron microscopy, and the right panel is its schema (magnification, x1,800). Invaded tumor cells (yellow) in an isolated and clustered form were observed within the altered layers of elastic (blue) and collagen (red) bundles. The intimal mesh-like structure of collagen fibers (red) had ruptured and disappeared in the vein.

difference was statistically significant $(\mathrm{P}=0.0054$, Fisher's exact test). MMP-7 expression was not significantly associated with tumor budding, lymphatic invasion, histological differentiation, growth type, morphology, DR on the superficial layer, depth of invasion, the Kudo's classification of degree of submucosal invasion, or the MM grade (17).

Ultrastructural alteration of venous invasion as determined by $L V$-SEM. Alterations of veins were examined by Victoria Blue B staining, collagen IV staining and LV-SEM, and the findings were compared (Fig. 3A-H). Normal veins were stained as a two-layered structure by collagen IV and Victoria Blue B staining. The structure of collagen IV staining was inside the veins, and exhibited a strong and thick linear pattern (Fig. 3A and C). Ultrastructural alterations of the veins were examined by LV-SEM. Normal veins were characterized by smooth bundles of elastic fibers in the adventitia and a mesh-like structure of collagen fibers in the intima (Fig. 3E and G). In samples with positive MMP-7 expression, venous invasion was frequently observed at the invasive front. In veins invaded by tumor cells, Victoria Blue B staining was thinner, and collagen IV staining was weaker, compared with normal veins (Fig. 3B and D). Under LV-SEM, veins invaded by tumor cells were characterized by an irregular surface that reflected the rupture of the two-layered structure of the vein (Fig. 3F). In addition, LV-SEM revealed that the thin part of collagen IV staining was characterized by partial disappearance of the mesh-like structure of collagen fibers in the intima. The invading tumor cells in the isolated and clustered form were visible within the altered layers of elastic and collagen bundles (Figs. $3 \mathrm{H}$ and 4).

\section{Discussion}

T1 CRC is the initial step in tumor spread, and may precisely reflect the metastatic potential of the primary tumor (19). The purpose of the present study was to investigate the association between MMP-7 expression at the invasive front and the risk factors of metastasis in T1 CRCs. A previous study on T1 CRCs (19) revealed that positive basilar and/or cytoplasmic MMP-7 immunostaining at the invasive front was significantly associated with the depth of invasion, tumor budding, lymphatic invasion and venous invasion. However, another study (20) indicated that the frequency of MMP-7 expression 
was significantly different among different histological grades, and that the frequency of MMP-7 expression at the invasive front differs depending on the selected microscopic field of the sections. Therefore, in the present study, MMP-7 immunostaining was evaluated by the median of the scores in five microscopic fields at the invasive front. Furthermore, objective discrimination between lymphatic and blood vessels was performed using immunostaining with an anti-D2-40 antibody, and double staining with H\&E and Victoria Blue B staining. Based on the above aspects, the present results revealed that the expression of MMP-7 was significantly associated with venous invasion in T1 CRCs. In a prospective surveillance program for early-stage CRC, venous invasion was a risk factor with a significant effect on disease-free survival, overall survival and disease recurrence (local or distant) (22). In addition, using LV-SEM, the present study observed morphological changes of collagen IV in veins. These findings may be histologically plausible because MMP-7 has important roles in the degradation of the principal structural components of veins such as collagen IV (7). A previous study (23) also reported that tumor cells expressing MMP-9 (which, similarly to MMP-7, degrades collagen IV) have significant positive correlations with venous invasion and liver metastasis in pancreatic cancer.

A previous study has shown expression of MMP-2 and MMP-9 in the glomerular basement membrane (GBM), which primarily consists of collagen IV, in immunoglobulin $\mathrm{A}(\operatorname{Ig} \mathrm{A})$ nephropathy (24). Another study (25) reported that morphological and qualitative alterations of type IV collagen on the GBM in IgA nephropathy are characterized by fractures of the GBM in LV-SEM. Prior to the present study, upon demounting the sections, these were stained with Masson's trichrome staining, periodic acid-methenamine-silver or Platinum Blue staining, which contain heavy metals (namely phosphotungstic acid, silver and platinum, respectively). Based on those preliminary results, Masson's trichrome staining was considered as the most effective method for improving the observation of colonic samples by LV-SEM (data not shown). A benefit of LV-SEM was the examination of the same veins observed by double staining of MMP-7 and Victoria Blue B. Following examination by double staining, the same sections were stained with Masson's trichrome staining and then assessed by LV-SEM. In the present study, the changes observed with collagen IV and Victoria Blue B staining in the invaded venous walls were consistent with the ultrastructural findings characterized by the rupture and disappearance of the normal structure of collagen and elastic fibers of veins by LV-SEM. These findings may be associated with MMP-7 expression in tumor cells at the front of venous invasion.

A limitation of the present study should be acknowledged. The association between MMP-7 expression and tumor budding in the current study was not significant. Basolateral staining of MMP-7 tended to occur in the cytoplasm of the invading tumor cells in the isolated and clustered form at the invasive front. However, since only five fields were observed, it is possible that the frequency of MMP-7 expression was underestimated. A recent study (26) revealed an association between the function of MMP-7 and its intracellular localization. Basolateral localization of MMP-7 has been associated with cell adhesion molecules and basement membrane components of basolaterally located substrates of tumor cells, such as E-cadherin and collagen IV (7). Such degradation by MMP-7 may initiate cancer invasion (20).

In conclusion, MMP-7 expression by tumor cells at the invasive front is significantly correlated with venous invasion in T1 CRCs. This finding may provide important information to evaluate the invasive potential of T1 CRCs. The expression of MMP-7 would be clinically useful as a prognostic marker of additional therapies and/or close surveillance.

\section{Acknowledgements}

The authors thank Mrs. T. Nagai and Mr. Y. Sasaki, medical technicians, of the Department of Pathology, Showa University School of Medicine (Tokyo) for their valuable assistance with immunohistochemical analysis of tissue samples, and Mrs. N. Takenaka, illustrator, of Nikko memorial hospital (Hokkaido) for creating the figures.

\section{References}

1. Kobayashi N, Saito Y, Uraoka T, Matsuda T, Suzuki H and Fujii T: Treatment strategy for laterally spreading tumors in Japan: Before and after the introduction of endoscopic submucosal dissection. J Gastroenterol Hepatol 24: 1387-1392, 2009.

2. Beaton C, Twine CP, Williams GL and Radcliffe AG: Systematic review and meta-analysis of histopathological factors influencing the risk of lymph node metastasis in early colorectal cancer. Colorectal Dis 15: 788-797, 2013.

3. Suzuki T, Sadahiro S, Mukoyama S, Ishikawa K, Yasuda S, Tajima T, Makuuchi H and Murayama C: Risk of lymph node and distant metastases in patients with early invasive colorectal cancer classified as Haggitt's level 4 invasion: Image analysis of submucosal layer invasion. Dis Colon Rectum 46: 203-208, 2003.

4. Ueno H, Hashiguchi Y, Kajiwara Y, Shinto E, Shimazaki H, Kurihara H, Mochizuki $\mathrm{H}$ and Hase K: Proposed objective criteria for 'grade 3' in early invasive colorectal cancer. Am J Clin Pathol 134: 312-322, 2010.

5. Benizri EI, Bereder JM, Rahili A, Bernard JL, Vanbiervliet G, Filippi J, Hébuterne X and Benchimol D: Additional colectomy after colonoscopic polypectomy for T1 colon cancer: A fine balance between oncologic benefit and operative risk. Int J Colorectal Dis 27: 1473-1478, 2012.

6. Roeb E, Arndt M, Jansen B, Schumpelick V and Matern S: Simultaneous determination of matrix metalloproteinase (MMP)-7, MMP-1, -3, and -13 gene expression by multiplex PCR in colorectal carcinomas. Int J Colorectal Dis 19: 518-524, 2004.

7. Ii M, Yamamoto H, Adachi Y, Maruyama Y and Shinomura Y: Role of matrix metalloproteinase-7 (matrilysin) in human cancer invasion, apoptosis, growth, and angiogenesis. Exp Biol Med (Maywood) 231: 20-27, 2006.

8. Adachi Y, Yamamoto H, Itoh F, Arimura Y, Nishi M, Endo T and Imai K: Clinicopathologic and prognostic significance of matrilysin expression at the invasive front in human colorectal cancers. Int J Cancer 95: 290-294, 2001.

9. Watanabe T, Itabashi M, Shimada Y, Tanaka S, Ito Y, Ajioka Y, Hamaguchi T, Hyodo I, Igarashi M, Ishida H, et al: Japanese society for cancer of the colon and rectum (JSCCR) guidelines 2010 for the treatment of colorectal cancer. Int J Clin Oncol 17: $1-29,2012$.

10. Kudo S, Lambert R, Allen JI, Fujii H, Fujii T, Kashida H, Matsuda T, Mori M, Saito H, Shimoda T, et al: Nonpolypoid neoplastic lesions of the colorectal mucosa. Gastrointest Endosc 68 (4 Suppl): S3-S47, 2008.

11. Hamilton SR, Bosman FT, Boffetta P, Ilyas M, Morreau H and Nakamura SI: WHO classification of tumours of the digestive system. In: Carcinoma of the colon and rectum. Bosman FT, Carneiro F, Hruban RH and Theise ND (eds): International Agency for Research on Cancer (IARC), Lyon, pp134-146, 2010. 
12. Ueno H, Murphy J, Jass JR, Mochizuki H and Talbot IC: Tumour 'budding' as an index to estimate the potential of aggressiveness in rectal cancer. Histopathology 40: 127-132, 2002.

13. Ueno H, Mochizuki H, Hashiguchi Y, Shimazaki H, Aida S, Hase K, Matsukuma S, Kanai T, Kurihara H, Ozawa K, et al: Risk factors for an adverse outcome in early invasive colorectal carcinoma. Gastroenterology 127: 385-394, 2004.

14. Shimoda T, Ikegami M, Fujisaki J, Matsui T, Aizawa S and Ishikawa E: Early colorectal carcinoma with special reference to its development de novo. Cancer 64: 1138-1146, 1989.

15. Kudo S: Endoscopic mucosal resection of flat and depressed types of early colorectal cancer. Endoscopy 25: 455-461, 1993.

16. Kashida $\mathrm{H}$ and Kudo SE: Early colorectal cancer: Concept, diagnosis, and management. Int J Clinical Oncol 11: 1-8, 2006.

17. Miyachi H, Kudo SE, Ichimasa K, Hisayuki T, Oikawa H, Matsudaira S, Kouyama Y, Kimura YJ, Misawa M, Mori Y, et al: Management of T1 colorectal cancers after endoscopic treatment based on the risk stratification of lymph node metastasis. J Gastroenterol Hepatol 31: 1126-1132, 2016.

18. Masaki T, Matsuoka H, Sugiyama M, Abe N, Goto A, Sakamoto A and Atomi Y: Matrilysin (MMP-7) as a significant determinant of malignant potential of early invasive colorectal carcinomas. Br J Cancer 84: 1317-1321, 2001.

19. Kurokawa S, Arimura Y, Yamamoto H, Adachi Y, Endo T, Sato T, Suga T, Hosokawa M, Shinomura Y and Imai K Tumour matrilysin expression predicts metastatic potential of stage I (pT1) colon and rectal cancers. Gut 54: 1751-1758, 2005 .

20. Tamahashi U, Kumagai J, Takizawa T, Sekine M and Eishi Y: Expression and intracellular localization of matrix metalloproteinases in intraductal papillary mucinous neoplasms of the pancreas. Virchows Arch 453: 79-87, 2008.

21. Barros SS, Henriques ÁC, Pereira KM, de Medeiros AM, Galvão HC and Freitas Rde A: Immunohistochemical expression of matrix metalloproteinases in squamous cell carcinoma of the tongue and lower lip. Arch Oral Biol 56: 752-760, 2011.
22. Gilardoni E, Bernasconi DP, Poli S, Garancini M, Luperto M, Zucchini N, Bovo G, Totis M, Bugatti A and Gianotti L: Surveillance for early stages of colon cancer: Potentials for optimizing follow-up protocols. World J Surg Oncol 13: 260, 2015.

23. Cho K, Matsuda Y, Ueda J, Uchida E, Naito Z and Ishiwata $T$ : Keratinocyte growth factor induces matrix metalloproteinase-9 expression and correlates with venous invasion in pancreatic cancer. Int J Oncol 40: 1040-1048, 2012.

24. Sekiuchi M, Kudo A, Nakabayashi K, Kanai-Azuma M, Akimoto $\mathrm{Y}$, Kawakami $\mathrm{H}$ and Yamada A: Expression of matrix metalloproteinases 2 and 9 and tissue inhibitors of matrix metalloproteinases 2 and 1 in the glomeruli of human glomerular diseases: The results of studies using immunofluorescence, in situ hybridization, and immunoelectron microscopy. Clin Exp Nephrol 16: 863-874, 2012.

25. Masuda Y, Yamanaka N, Ishikawa A, Kataoka M, Arai T, Wakamatsu K, Kuwahara N, Nagahama K, Ichikawa K and Shimizu A: Glomerular basement membrane injuries in IgA nephropathy evaluated by double immunostaining for $\alpha 5$ (IV) and $\alpha 2$ (IV) chains of type IV collagen and low-vacuum scanning electron microscopy. Clin Exp Nephrol 19: 427-435, 2015.

26. Harrell PC, McCawley LJ, Fingleton B, McIntyre JO and Matrisian LM: Proliferative effects of apical, but not basal, matrix metalloproteinase-7 activity in polarized MDCK cells. Exp Cell Res 303: 308-320, 2005.

This work is licensed under a Creative Commons Attribution-NonCommercial-NoDerivatives 4.0 International (CC BY-NC-ND 4.0) License. 